University of Nebraska - Lincoln

DigitalCommons@University of Nebraska - Lincoln

Peter Dowben Publications

Research Papers in Physics and Astronomy

November 2005

\title{
Intra-atomic aspects of magnon-plasmon interactions
}

\author{
Ralph Skomski \\ University of Nebraska-Lincoln, rskomski2@unl.edu \\ Peter A. Dowben \\ University of Nebraska-Lincoln, pdowben@unl.edu
}

Follow this and additional works at: https://digitalcommons.unl.edu/physicsdowben

Part of the Physics Commons

Skomski, Ralph and Dowben, Peter A., "Intra-atomic aspects of magnon-plasmon interactions" (2005). Peter Dowben Publications. 4.

https://digitalcommons.unl.edu/physicsdowben/4

This Article is brought to you for free and open access by the Research Papers in Physics and Astronomy at DigitalCommons@University of Nebraska - Lincoln. It has been accepted for inclusion in Peter Dowben Publications by an authorized administrator of DigitalCommons@University of Nebraska - Lincoln. 


\title{
Intra-atomic aspects of magnon-plasmon interactions
}

\author{
R. Skomski ${ }^{\mathrm{a})}$ and P. A. Dowben \\ Center for Materials Research and Analysis, 111 Brace Laboratory, P.O. Box 880111, \\ University of Nebraska, Lincoln, Nebraska 68588 \\ and Department of Physics and Astronomy, 116 Brace Laboratory, P.O. Box 880111, \\ University of Nebraska, Lincoln, NE 68588
}

(Presented on 1 November 2005; published online 28 April 2006)

\begin{abstract}
Magnon-plasmon interactions are modeled by considering the spin-dependent dielectric response of atoms placed in crystalline environment. Hund's exchange rules favor parallel spin alignment, but the strength of the exchange depends on the displacement of the centers of gravity of the atomic spin-up and spin-down electron charge clouds. The intra-atomic exchange is modeled by considering a Hubbard-type interaction, and interatomic interaction then yields a $k$-space dispersion. The eigenmodes of the plasma are a mixture of spin-up and spin-down degrees of freedom, described by a $2 \times 2$ interaction matrix. Minority and majority bands yield different plasmon frequencies. However, these modes are not orthogonal but coupled by intra-atomic exchange and obtained by explicit matrix diagonalization. The effect is largest for small wave vectors, in agreement with experiment. (C) 2006 American Institute of Physics. [DOI: 10.1063/1.2177070]
\end{abstract}

\section{INTRODUCTION}

Interactions between magnetic and electric degrees of freedom have been investigated very extensively, but magnon-plasmon interactions, without any coupling through phonons, are almost never considered. ${ }^{1-3}$ The main reason is that the effect is quite small. Magnon energies tend to be much smaller than plasmon energies, so that the experimental investigations of the interactions are a challenge. An exception is dilute magnetic semiconductors, such as $\mathrm{Eu}_{1-x} \mathrm{Gd}_{x} \mathrm{O},{ }^{2}$ where low carrier densities yield low plasmon energies. Recently, spin-polarized electron energy loss spectroscopy (SPEELS) has been used to investigate magnonplasmon interactions in thin films of strained $\mathrm{Gd}(0001)$ on $\mathrm{Mo}(112){ }^{4}$ In this technique, a spin-polarized electron is scattered off the surface at remanence, and the energy of the scattered electrons is analyzed to determine the loss structure. ${ }^{5}$ The experiment indicates spin-polarized plasmons, in spite of the high concentration of $5 d$ and $6 s$ conduction electrons in $\mathrm{Gd}$.

In this paper, we investigate spin-dependent plasmon modes in nonsemiconducting systems with high plasmon frequencies. The considered excitations refer to longitudinal spin degrees of freedom, as contrasted to ordinary magnons, which are transversal. The difference is that longitudinal modes involve intra-atomic exchange energies larger than about $1 \mathrm{eV}$, whereas transversal magnon modes are due to interatomic exchange of less than about $0.1 \mathrm{eV}$. In other words, the present excitations involve changes in the magnitude of the local moment, as compared to moment rotations that change $S_{z}$ but keep $S(S+1)$ fixed.

\section{BASIC MECHANISM}

In the simplest case, the plasmon is created by an inhomogeneous magnetic field acting on a spin-polarized mag-

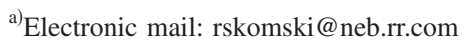

netic body. If the position of the magnet is fixed, the field creates a plasmon mode and electric surface charges. Figure 1 illustrates the phenomenon for an inhomogeneous magnetic field $\mathbf{H}=H(x) \mathbf{e}_{z}$ characterized by a positive magnetization gradient $\partial H / \partial x$. Since the Zeeman interaction of a moment $\mathbf{m}$ with the external field is equal to $-\mu_{0} \mathbf{m} \cdot \mathbf{H}$, the moment-carrying electrons move towards regions of higher field intensity. This corresponds to a plasmon mode of some magnitude or electron displacement. For laboratory-scale magnetic fields, the effect is relatively small, because the Zeeman energy competes against much stronger energies of electrostatic origin. The relative strength of the magnetic interactions is of the order of $\alpha^{2}{ }^{6}$, where $\alpha=1 / 137$ is Sommerfeld's fine-structure constant. By contrast, SPEELS experiments correspond to much larger local exchange fields.

In a free-electron picture, a spin-polarized electron gas gives rise to different plasmon frequencies $\omega_{\uparrow} \sim n_{\uparrow}^{1 / 2}$ and $\omega_{\downarrow}$ $\sim n_{\downarrow}^{1 / 2}$, which can be probed experimentally. However, free electrons are a poor starting point, because atomic magnetic moments are centered around the atomic cores, even in itin-

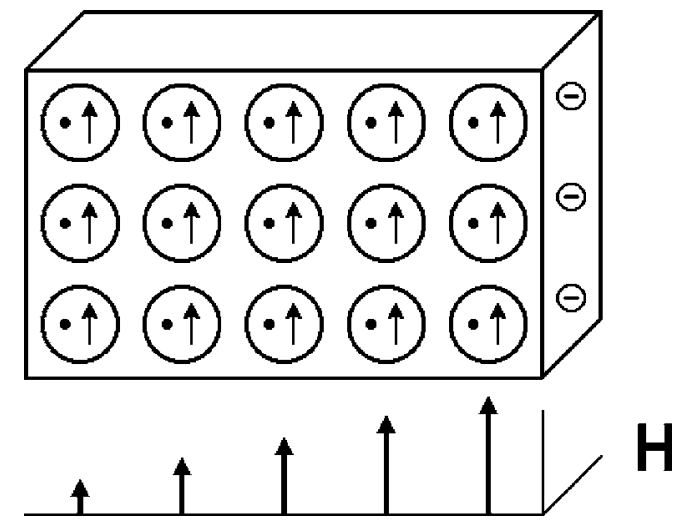

FIG. 1. Plasmon created by an inhomogeneous magnetic field $H$ acting on a spin-polarized magnetic body. If the position of the body is fixed, the field creates a plasmon mode and electric surface charges. 


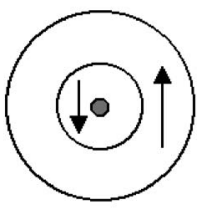

(a)

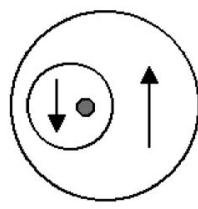

(b)

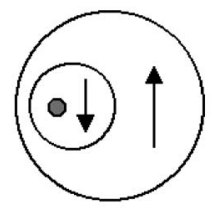

(c)
FIG. 2. Magnetic phonon-electron interaction model: (a) ground state, (b) out-of-phase excitation, and (c) in-phase excitation. The small gray circle is the atomic core (nucleus).

erant magnets. In $\mathrm{Gd}$, the magnetic moment is caused by localized $4 f$ electrons, but the Gd $4 f$ electron creates an exchange field that slightly polarizes the $5 d$ and $6 s$ conduction electrons of the $\mathrm{Gd}$ and gives rise to different electron densities $n_{\uparrow}$ and $n_{\downarrow}$.

\section{CALCULATION AND RESULTS}

Let us now investigate the intra-atomic aspect of the magnon-plasmon interactions. Each atom (index $i$ ) has two subshells, $\uparrow$ and $\downarrow$, and these shells may be displaced with respect to the position $\mathbf{R}_{i o}$ of the atomic core. Let us denote the displacements by $\mathbf{R}_{i \uparrow}$ and $\mathbf{R}_{i \downarrow}$. Figure 2 illustrates that there are two types of excitations, out-of-phase excitations where $\mathbf{R}_{i \uparrow}$ and $\mathbf{R}_{i \downarrow}$ point in different directions (b) and inphase excitations where $\mathbf{R}_{i \uparrow}$ and $\mathbf{R}_{i \downarrow}$ point in the same direction (c). We treat these excitations in a harmonic approximation and assume that the shape of the $\uparrow$ and $\downarrow$ shells remains unchanged (rigid orbitals). Next, we expand the magnetic energy into powers of the small vectors $\xi_{i \uparrow}=\mathbf{R}_{i \uparrow}-\mathbf{R}_{i o}$ and $\xi_{i \downarrow}=\mathbf{R}_{i \downarrow}-\mathbf{R}_{i o}$. Here we ignore anisotropic effects, which would mix different displacement directions, and consider the longitudinal modes $\xi_{i \uparrow}$ and $\xi_{i \downarrow}$ shown in Fig. 2.

The procedure is illustrated by considering the Coulomb energy $E_{C}$, which is the origin of the intra-atomic exchange. In the Hubbard formulation, $E_{C}=U n_{\uparrow} n_{\downarrow}$, where $n_{\uparrow}$ and $n_{\downarrow}$ are electron densities, ${ }^{7,8}$ but in the present case, a more general formulation of this expression must be used,

$$
E_{C}=\int U\left(\mathbf{r}-\mathbf{r}^{\prime}\right) \rho_{\uparrow}\left(\mathbf{r}-\mathbf{R}_{i \uparrow}\right) \rho_{\downarrow}\left(\mathbf{r}^{\prime}-\mathbf{R}_{i \downarrow}\right) d \mathbf{r} d \mathbf{r}^{\prime}
$$

Here $\rho_{\uparrow}(\mathbf{r})=\psi_{\uparrow} *(\mathbf{r}) \psi_{\uparrow}(\mathbf{r})$ and $\rho_{\downarrow}(\mathbf{r})=\psi_{\downarrow} *(\mathbf{r}) \psi_{\downarrow}(\mathbf{r})$ are electron densities. This expression can be evaluated by expansion into powers of $\xi_{i \uparrow}$ and $\xi_{i \downarrow}$. The same is true for other energy contributions, such as the attractive interaction between the atomic core and the $\uparrow$ and $\downarrow$ shells. The resulting total on-site energy is

$$
E=E_{0}+\frac{u \uparrow}{2} \xi_{\uparrow}^{2}+u_{m} \xi_{\uparrow} \xi_{\downarrow}+\frac{u \downarrow}{2} \xi_{\downarrow}^{2}
$$

In addition to this on-site energy, there are wave-vector dependent contributions. Figure 3 illustrates this by comparing two modes with (a) $k=0$ and (b) $k=\pi / a$. The energy of Fig. 4(b) is higher than that of Fig. 4(a), because in (b) the $\uparrow$ orbitals nearly overlap. The wave-vector dependence of the modes shown in Fig. 4 refers to the $\uparrow$ electrons. In the figure, the $\downarrow$ electron clouds are all shifted to the right direction. However, there may also be other modes, such as $\downarrow$ and

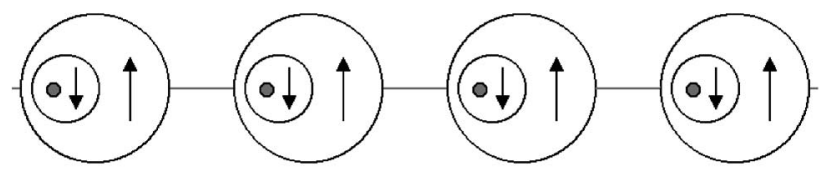

(a)

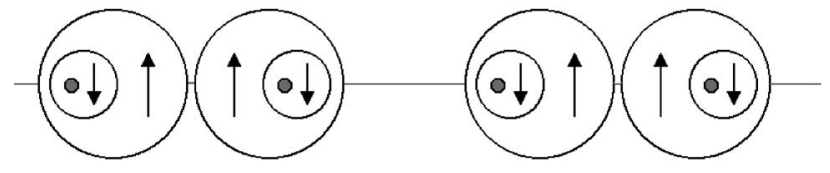

(b)

FIG. 3. Wave-vector dependence: (a) one $k=0$ mode and (b) one $k=\pi / a$ mode.

mixed modes, where $\uparrow$ and $\downarrow$ electrons on different atoms interact with each other.

The eigenfrequencies $\omega$ of the excitations are obtained by diagonalizing the $2 \times 2$ matrix expression

$$
\left(\begin{array}{cc}
\omega^{2} & 0 \\
0 & \omega^{2}
\end{array}\right)=\left(\begin{array}{cc}
\omega_{\uparrow}^{2}(\mathbf{k}) & \omega_{m}^{2}(\mathbf{k}) \\
\omega_{m}^{2}(\mathbf{k}) & \omega_{\downarrow}^{2}(\mathbf{k})
\end{array}\right) .
$$

In the simple case of a chain of magnetic atoms, the wavevector dependence is given by $1-\cos k a$. Figure 4 shows a typical $k$ dependence for this one-dimensional example. A general feature, not restricted to the one-dimensional model, is some admixture of $\downarrow$ character to the $\uparrow$ modes. However, this mixing refers to the origin of the mode. The magnitude of the local magnetization changes, because the electron clouds of opposite spin are shifted to different positions, but the mxing does not indicate spin flipping. In fully spinpolarized magnets, the dielectric response is provided by $\uparrow$ electrons, which then behave similar to ordinary electrons.

\section{DISCUSSION AND CONCLUSIONS}

The modes described in Sec. III mean that $\uparrow$ and $\downarrow$ electron clouds have different properties. Equation (2) and (3) parametrize this difference, but no attempt is made in this paper to calculate the parameters from first principles. For example, $\omega_{\uparrow}, \omega_{\downarrow}$, and $\omega_{m}$ depend on the interatomic hopping

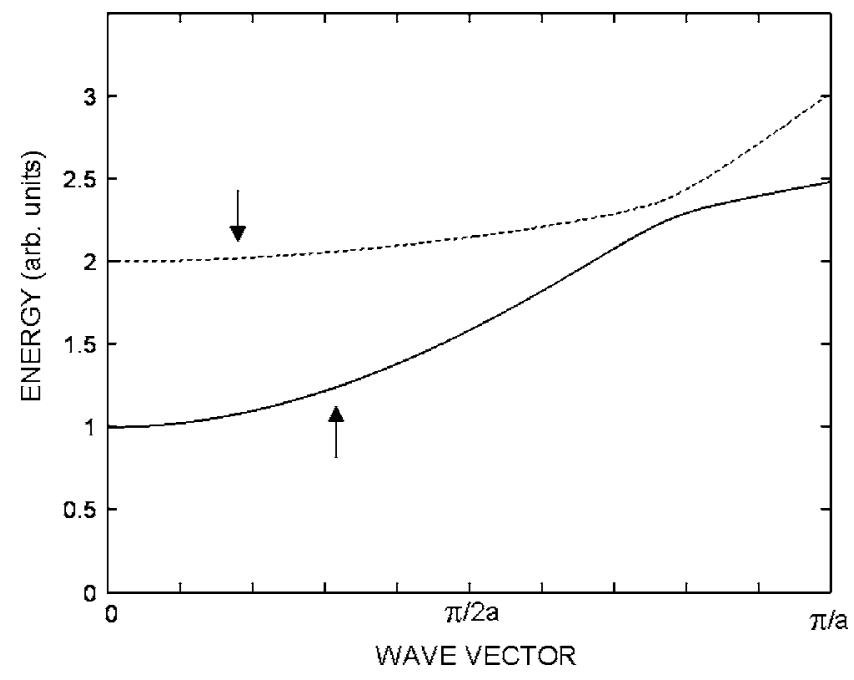

FIG. 4. Wave-vector dependent modes. 
integrals, which determine, in combination with Eq. (1), the ferromagnetic moment of itinerant magnets. The harmonic approximation used in Eq. (2) is reasonable, because the effect is not very large. ${ }^{4}$ Another relatively unessential approximation is the assumption of rigid charge clouds.

An experimental challenge is the small magnitude of the effect, and there is no hope to observe excitations such as those shown in Fig. 2 in laboratory-scale field gradients of the order of $1 \mathrm{~T} / \mathrm{cm}$. The SPEELS experiments outlined in Ref. 4 involve much higher energies, because the spinpolarized electrons interact directly with the $\uparrow$ and $\downarrow$ electron clouds. As emphasized in Sec. II, this corresponds to much larger effective local magnetic fields (exchange fields). Figure 4 shows that the excitations are accessible most easily at small wave vectors, in agreement with the preliminary experimental findings. Naturally, the relatively simple model of Sec. III captures only the most basic features of the phenomenon, and refined materials-specific calculations of this interesting effect remain a challenge for future research.

In conclusion, we have analyzed magnetic excitations of intra-atomic origin. They are related to ordinary magnons but involve longitudinal magnetic degrees of freedom and involve much higher energies. This makes it possible to excite them in metallic ferromagnets, as contrasted to magnetic semiconductors. The effect is wave vector dependent and can be investigated experimentally by spin-polarized electron energy loss spectroscopy.

\section{ACKNOWLEDGMENTS}

This work was supported by the National Science Foundation through the NSF "QSPINS" MRSEC (DMR0213808) and by the Center for Materials Research and Analysis at the University of Nebraska, which is funded by the Nebraska Research Initiative.

${ }^{1}$ D. J. Kim, Phys. Rep. 171, 129 (1988).

${ }^{2}$ G. Baskaran and K. P. Sinha, Pramana: Journal of Physics 1, No. 1, 31 (1973).

${ }^{3}$ V. G. Baryakhtar, F. G. Bass, and Z. Z. Makhumdov, Sov. Phys. Solid State 12, 1596 (1971)

${ }^{4}$ H.-K. Jeong, R. Skomski, D. Wisbey, and P. A. Dowben, Phys. Lett. A 341, 508 (2005)

${ }^{5}$ Spin-dependent plasmons and small intraband excitations across the Fermi level do not involve a spin flip scattering, so that spin detection after the scattering event is not necessary if a spin polarized source is used.

${ }^{6}$ R. Skomski, H.-P. Oepen, and J. Kirschner, Phys. Rev. B 58, 3223 (1998).

${ }^{7}$ W. Jones and N. H. March, Theoretical Solid State Physics (Dover, New York, 1985), Vol. 1.

${ }^{8}$ R. Skomski and J. M. D. Coey, Permanent Magnetism (IOB, Bristol, 1999). 